\title{
Fetal Cardiac Therapy: Are We There Yet?
}

\author{
Ra-id Abdulla
}

Published online: 27 June 2014

(C) Springer Science+Business Media New York 2014

Recently, after attending a fetal cardiology symposium, I wondered if the advancement in this field has plateaued. The capabilities of echocardiographic equipment continue to improve, though at a gentle slope and the application of fetal cardiac intervention continues to be either technically difficult or dogged by limited benefits. Furthermore, the diagnosis and treatment of fetal arrhythmias continues to be the same it has been 10-15 years ago. With the tremendous advancement in fetal cardiac imaging, I have no doubt that fetal patients diagnosed in this era receive care not provided to them in past decades, nevertheless, the improvement is not vast leaving one to question why have we not seen steeper improvement in our knowledge and practice of fetal cardiology. Another manifestation of the slower pace of progress in this field could be ascertained through examining the frequency with which articles in this field are referenced. The relative decline in article referencing in medical publications in this field when compared to a much steeper increase in referencing articles in the field of pediatric cardiac interventional procedures suggests a decline in the rate of growth of research in this field.

Perhaps a significant factor tempering advancement in this field is that the overwhelming majority of congenital heart diseases discovered in the second and third trimesters of gestation do not cause much undue hemodynamic burden on the fetus. It is not until this circulation is altered through regurgitation or other rare pathologies that there are unwanted hemodynamic changes leading to fetal morbidity and mortality. Even fetal interventions proposed to alter the evolution of fetal heart disease such as in

R. Abdulla $(\bowtie)$

Department of Pediatric Cardiology, Rush University Medical Center, Chicago, IL 60612, USA

e-mail: ra-id_abdulla@rush.edu hypoplastic left heart syndrome with intact atrial septum or critical aortic stenosis with a left ventricle lagging in growth remain technically challenging with limited data and questionable long- term benefit.

Therefore, in view of stable in utero status combined with limited improvement of outcomes from fetal cardiac intervention, one is forced to accept the observed pathology and limit efforts to delivery and what ensues thereafter. This is most probably aggravated by the limited number of clinical cases warranting intervention and the limited experimental animal research projects in this field. The latter may be limited in its breadth because of high cost and the impression that it will not significantly improve the in utero course of disease. Animal research many decades ago provided us with valuable understanding of fetal cardiovascular physiology and pathophysiology through Dr. Rudolph's pioneering work on fetal lambs. More recently, research on potential therapeutic applications of ultrasound technology in creating septal defects when indicated suggests that fetal intervention in congenital heart disease may indeed one day be standard practice once the tools and methodologies are made available to us. Clearly, much more research and experimentation is needed before this becomes available to clinical practice.

Perhaps significant advances in fetal cardiac intervention is not possible until imaging capabilities can show fetal cardiovascular development during early parts of the first trimester. The ability to visualize abnormal development of cardiac structures as they occur during the 4th to 8th week of life, a feat difficult to imagine possible today, will certainly provide better understanding of how congenital heart disease evolves and consequently precipitate immense growth in fetal cardiac intervention at a much earlier stage. Advances in imaging and technical instrumentation would open the door for earlier intervention, 
potentially changing the evolution of congenital heart disease in the fetus. Such intervention may be through genetic and pharmacological means leading to earlier cardiovascular remodeling rather than through the use of brute force.

Another hindering factor in the development of this field is the lack of a clearly defined body of experts to advocate for research, development and education. Over the past decades there have been numerous high caliber meetings dedicated to present new discoveries in this field similar to the one I recently attended in Chicago through Rush University. I believe this process can be further advanced through the creation of a fetal cardiology organization which will allow experts in this field to share resources as they advocate for continuation and expansion in clinical as well as basic science research in fetal cardiology. An organization of this nature can allow the organizers of fetal cardiology meetings to share a pool of speakers and other resources, create an advocacy and support system for research requiring significant funding and coordinate efforts with specialists in maternal fetal medicine and interventional cardiology for the betterment of fetal cardiac care.

So, are we there yet? Not by any stretch of the imagination, but the challenge to get there will be much fun! 